\title{
ANALYSIS OF THE CARBON FOOTPRINT OF COASTAL PROTECTION SYSTEMS
}

\author{
A.L. Labrujere ${ }^{1}$ and H.J. Verhagen ${ }^{2}$
}

\begin{abstract}
When calculating the Carbon Footprint for a product or service, a direct link is made between the total amount of consumed energy and the produced amount of carbon dioxide during production. For that reason calculating the carbon footprint of various alternatives is a very straightforward method to compare energy consumption and more importantly environmental pollution. Applying this method to large hydraulic engineering projects is not being done frequently. In this study the possibilities to apply the Carbon Footprint method to coastal protection systems have been explored and analyzed. The analyses are based on a case study: A reinforcement work at the Dutch coast.
\end{abstract}

Keywords: Carbon Footprint; coastal protection; carbon dioxide; energy

\section{INTRODUCTION}

\section{Carbon Footprint}

Each product or service is responsible for a certain amount of $\mathrm{CO}_{2}$ emissions. To compose the Carbon Footprint of a product, according to the LCA methodology all carbon contribution for the production should be accounted for. This includes the supply of raw materials, transport, construction activities, maintenance works and disposal. Each activity produces an amount of $\mathrm{CO}_{2}$ emission, caused by energy consumption during the activity. The summation of all the $\mathrm{CO}_{2}$ emissions is called the Carbon Footprint. If there are $n$ (number) activities consuming energy during the lifetime of a product, each causing a specific amount of $\mathrm{CO}_{2}\left(e_{t o t, i}\right)$ per quantity, the Equation (1) for the Carbon Footprint (CF) will be:

$$
C F=\sum_{i=1}^{n} e_{t o t, i} \cdot \text { quantity }_{i}
$$

The quantity in which the emission coefficient is expressed can vary from activity to activity. In transport for instance this coefficient is normally expressed in $\mathrm{kg} \mathrm{CO}_{2} / \mathrm{km}$ where for the production of raw materials this parameter is normally expressed in $\mathrm{kg} \mathrm{CO}_{2} / \mathrm{kg}$ or tonnes. The outcome of the $\mathrm{CF}$ is always an amount of $\mathrm{CO}_{2}$ expressed in weight $(\mathrm{kg})$.

\section{Carbon Footprints in Coastal Engineering}

Not much research has been done on the application of the Carbon Footprint in coastal engineering. A study on the carbon emissions of breakwaters by Bruce and Chick (2009) is one of the most striking examples. A result from this is that there is a low comprehension as to the most sustainable solution in terms of carbon pollution.

In order to bring down the total amount of emitted carbon, products with a relatively lower Carbon Footprint should be promoted. Reducing carbon dioxide emissions is seen as essential for sustaining the environment.

\section{Boundaries}

The Carbon Footprint represents the legacy that the product leaves behind on the earth. When a Carbon Footprint is determined a clear starting and ending point in time should be defined in order to determine a representative legacy. Double counting and the incorporating of emissions in the footprint that are strictly not contributing to a product should be avoided. The start end ending point are the boundaries of the Carbon Footprint and will determine the number ( $n$ ) of activities in Equation (1).

Normally the design lifetime of a product is a solid basis of comparison. When a product is disposed (or reused) at the end of its lifetime the entire Life Cycle is a better choice. This is done in for instance a Life Cycle Analysis (LCA).

Especially in the case of projects as regarded in this study boundaries are very important. In case of a coastal reinforcement by means of beach nourishment, the product is not constructed from scratch.

\footnotetext{
${ }^{1}$ Ministry of Infrastructure and Environment, the Directorate General for Public Works and Water Management, Centre for Water Management, Zuiderwagenplein 2, 8224 AD Lelystad, The Netherlands

2 Delft University of Technology, Section Hydraulic Engineering, Faculty of Civil Engineering and Geosciences (CiTG), Stevinweg 1, 2500 GA Delft, The Netherlands
} 
The beach and a current protection are already present and contribute to the end result. Disposal of the protection works at the end of its lifetime is not likely, however (an additional) reinforcement in time is.

This is different than for instance in the production of a car. A car is build for raw materials and disposed at the end of its lifetime. Therefore limits (in terms of time) should be established on forehand when determining the Carbon Footprint. Only then a clear comparison between alternatives can be made.

\section{CASE STUDY}

\section{Scheveningen}

In this study several designs for a coastal reinforcement, based on different materials, are judged on their environmental impact. The basis of the comparison are the amounts of embodied carbon (Carbon Footprint) of different designs. The different materials all require their own transport en placement method. Each step contributes to the total amount of carbon as illustrated in the introduction. The result is an emitted amount of Carbon Dioxide per running meter of coastal reinforcement

A case study is the chosen method of comparison because it ensures a constant set of boundary conditions for each design. The chosen location also determines the transportations distances for the construction materials for delivery at the project site.

The location which is chosen for this analysis is Scheveningen, it is a well known beach area and it is located in a part of the Netherlands that is fairly well accessible to inland shipping and transport by road.

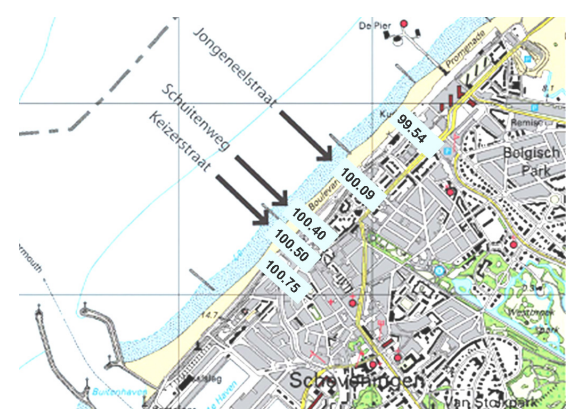

Figure 1 Location Scheveningen and adjacent transport systems at the Dutch coast

\section{Weak link}

Scheveningen (Figure 1) is part of the Dutch primary sea defense system and reinforcement works are being undertaken at the location. The reinforcement is being executed because it is one of the weak links in the Dutch coastal system. This location and a few other locations at the Dutch coast are not meeting the Dutch safety requirements.

Several preliminary designs were taken into account in the reinforcement of Scheveningen. All designs are aimed to ensure the safety of the hinterland of Scheveningen. The safety can be achieved either by means of a soft or hard solution.

Soft solutions have been applied at Scheveningen in the past. Several beach nourishments have been carried out in past decades and have kept the beach of Scheveningen at the required width. Sand will in course of time not only be redistributed in the cross direction but will also be transported along the shore in mostly Northern direction. Soft solutions are not permanent and will always need maintenance work in the form of additional nourishments.

For that reason a hard solution is the actual chosen alternative and in this study is regarded in different forms as well. The lifetime of both soft and hard solution is defined equally as 50 years.

\section{Alternatives}

In this study 5 different alternatives are compared. The five alternatives include the following soft and hard solutions. In the design of the alternatives the primary sea defense and the components that contribute to that function are solely regarded. Other functions as recreational or esthetical have been disregarded.

-Beach nourishment. This alternative entails a large starting nourishment and several maintenance nourishment over the course of time (50 years). For the nourishments a big 
hopper dredger is considered corresponding the vessel that is generally used for this type of activities.

- Concrete columns. This design is based on a top layer of concrete columns with a variation of columns heights $(0,45 ; 0,40$ and $0,25 \mathrm{~m})$ and concrete density $\left(2900\right.$ and $\left.2300 \mathrm{~kg} / \mathrm{m}^{3}\right)$. The columns are placed on top of filter layer and a geotextile

-Quarry stone. In this alternative a top layer of 0,3 to 1 tonne stones is designed. A filter layer, toe protection and geotextile are also accounted for.

-Asphalt top layer. In this design a top layer of hydraulic asphalt is places on top of a filter layer.

-Elastocoast. This is a relatively new and experimental solution for coastal protection top layers. The solution consists of a Polyurethane bounded aggregate (PBA) layer. This is placed on a filter layer with a similar layer thickness on top of a geotextile.

Because the beach, due to natural processes, will erode for all hard constructions (concrete columns, quarry stone, asphalt and elastocoast) a small additional beach nourishment is incorporated in the designs. The general cross section of each of the alternatives (hard solutions) is chosen to be similar and as simple as represented in the figure 2 . The outer slope of all designs for hard solutions is set at $1: 3$.

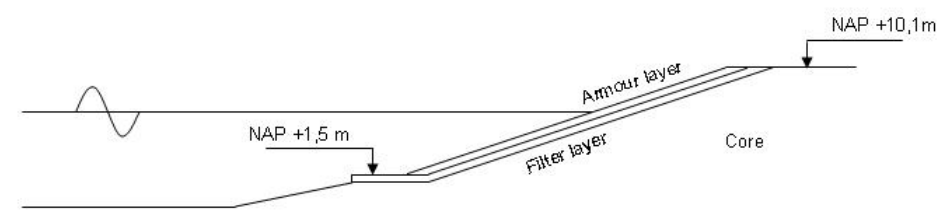

Figure 2 General cross section for the hard solutions (concrete columns, quarry stone, asphalt and elastocoast)

\section{Total amounts of material for the alternatives}

The total amounts of beach nourishment comes down to $1786 \mathrm{~m}^{3} / \mathrm{m}$. Of this amount roughly $1 / 3$ is applied at the start of the project, the remaining part is applied during the lifetime (50years) of the solution as maintenance activities.

An additional small nourishment that is taken into account for the hard solutions is estimated at $400 \mathrm{~m}^{3} / \mathrm{m}$ over 50 years. Other quantities for the hard solutions are given in Table 1 .

\begin{tabular}{|c|c|c|c|c|c|c|c|}
\hline & \multicolumn{2}{|c|}{$\begin{array}{l}\text { Concrete } \\
\text { columns }\end{array}$} & \multicolumn{2}{|c|}{ Quarry stone } & \multirow{2}{*}{$\begin{array}{l}\text { Asphalt } \\
\mathrm{kg} / \mathrm{m} \\
\end{array}$} & \multicolumn{2}{|c|}{ Elastocoast } \\
\hline & $\mathrm{m}^{3} / \mathrm{m}$ & $\mathrm{m}^{2} / \mathrm{m}$ & $\mathrm{kg} / \mathrm{m}^{2}$ & $\mathrm{~kg} / \mathrm{m}$ & & $\mathrm{kg} / \mathrm{m}^{2}$ & $\mathrm{~kg} / \mathrm{m}$ \\
\hline $\begin{array}{l}\text { Concrete columns } \\
\text { Toe wall } \\
\text { Concrete strips } \\
\text { Filter layer } \\
\text { Second filter layer } \\
\text { Geo Textile } \\
\text { Wash material } \\
\text { Core } \\
\text { Quarry stone armour layer } \\
\text { Toe protection } \\
\text { Asphaltic top layer } \\
\text { PBA }\end{array}$ & $\begin{array}{l}15,58 \\
1,48 \\
0,19 \\
3,49 \\
9,51 \\
0,67 \\
30\end{array}$ & 39.37 & $\begin{array}{l}850 \\
0,51 \\
\\
1325 \\
850\end{array}$ & 79500 & $\begin{array}{l}79500 \\
1150 \\
1150\end{array}$ & $\begin{array}{l}648 \\
0,51 \\
644\end{array}$ & 79500 \\
\hline
\end{tabular}

\section{EMISSIONS}

\section{Greenhouse gasses}

By using energy sources containing carbon such as coal, petroleum and natural gas, several gases are produced and launched into the atmosphere. Phenomena like the greenhouse effect and an increase in global average temperatures have been linked to the quantities of these gasses that have been emitted into the atmosphere in the past century.

The gasses that are considered to have the largest impact on the environment are carbon dioxide $\left(\mathrm{CO}_{2}\right)$, methane $\left(\mathrm{CH}_{4}\right)$, nitrous oxide $\left(\mathrm{N}_{2} \mathrm{O}\right)$ gases and fluorine containing gasses $(\mathrm{HFC}, \mathrm{SF} 6, \mathrm{PFC})$ 
(Hendriks, 2001). They are summarized in the term greenhouse gasses and from them carbon dioxide has shown to have the largest impact. Therefore other greenhouse gasses are often expressed in Carbon Dioxide Equivalent (CDE) emissions in order to add them up to a total (Table 2). They are also expressed as $\mathrm{CO}_{2} \mathrm{e}$ or $\mathrm{CO}_{2} \mathrm{eq}$.

\begin{tabular}{|l|l|l|}
\hline \multicolumn{3}{|l|}{ Table 2. $\mathbf{C O}_{2}$ equivalent emissions (IPCC, 2001) } \\
\hline Species & Chemical formula & $\mathrm{CO}_{2} \mathrm{e}$ \\
\hline Carbon Dioxide & $\mathrm{CO}_{2}$ & 1 \\
Methane & $\mathrm{CH}_{4}$ & 23 \\
Nitrous oxide & $\mathrm{N}_{2} \mathrm{O}$ & 296 \\
HFC's & - & $12-12000$ \\
Sulphur hexafluoride & $\mathrm{SF}_{6}$ & 22200 \\
PFC's & - & $5700-11900$ \\
\hline
\end{tabular}

\section{Scope}

The scope determines the boundary conditions for the consumed energy that is accounted for. When the energy of an entire life cycle of a product is summed up, the scope is 'Cradle-to-Grave'. It includes the total amount of energy from manufacturing, transport, energy to manufacture capital equipment, heating and lighting of factory, maintenance and disposal.

That total amount of energy is also called the embodied energy. The emissions that follow from the embodied energy are called the embodied carbon. These two values (embodied energy and embodied carbon) can vary as the scope of the footprint is changes. When the embodied energy and carbon are determined up to the point where the product leaves the factory gate this is known as 'Cradle-to-Gate'.

The scope which includes all of the energy consumed until the product has reached is point of use is known as 'Cradle-to-Site'.

\section{Reuse}

Apart from the before mentioned three methods to determine the embodied carbon a fourth variation incorporates the recycling of products. In this method total primary energy consumed (carbon released) over its life cycle is determined including the steps at the end of the cycle to prepare the material for a new life in a new product. It is called 'Cradle-to-Cradle'.

This principle is a variation on the two more general methods to approach the reuse of material in Carbon Footprinting (ICE database, 2008).

- Recycled content approach: This is a method that credits recycling. It is a 'Start of life' method in which the amount of material that is recycled is credited. If for instance $40 \%$ off all the metal used for a products is recycled used, then the recycled content is $40 \%$. A reduction of $40 \%$ of the emissions from metal can be accounted for.

-Substitution method: This method credits recyclability. The recycled content is not considered here. Instead the ability for materials to be recycled at the end of the products lifetime is evaluated. For instance for metals the recyclability could be taken as $85 \%$, indicating that this amount of the metal will be prepared for reuse after the lifetime of the original product. The recyclability will therefore reduce the emissions taken into account for metal for the Carbon Footprint of the current product.

\section{Direct and indirect emissions}

Furthermore Greenhouse Gas (GHG) emissions can be divided into direct and indirect emissions. The Greenhouse Gas protocol is a worldwide accepted method to determine emissions. The GHG protocol (2004) defines direct emissions as emissions from sources that are owned or controlled by the producing company.

And indirect emissions are defined as a consequence of the activities of the producing company, but occur at sources owned or controlled by another companies. In this study only direct emissions are accounted for.

\section{Emission coefficients}

Emission Coefficients are not dimensionless as their name would suspect. They merely give a linear relation between a material or activity and the corresponding emission (e.g. $\mathrm{CO}_{2} / \mathrm{kg}$ or $\mathrm{CO}_{2} / \mathrm{m}^{2}$ ). 


\section{LCA's and databases}

Environmental impacts are normally based on Life Cycle Analyses (LCA). LCA is an internationally standardized method for the evaluation of the environmental burdens and resources consumed during the life cycle of a product.

There are several companies producing LCA's for many different products and activities. The analysis includes pollutions to air, water and ground. This study focused only on air pollution through $\mathrm{CO}_{2}$ emissions. Other initiatives are set up to collect these LCA's and present information from them in well organized databases.

Databases concerning data on $\mathrm{CO}_{2}$ emissions are available because of the globally spread intentions to reduce the total national amount of emissions. Resulting from the specific setup of the database each has its own area of specialization.

\section{Energy split}

Generally there is not one single energy source but several fuel types involved in the production of one product. This makes the emission coefficients of energy generation even more complicated and important. An overview of the distribution of the embodied energy over the different energy sources for a product or activity is known as the fuel split. It indicates which part of the energy consumption was generated by which energy source.

\section{Emissions for energy generations}

When $n$ number of fuel sources are consumed for the production the total emission (Equation 2) of a product is calculated as follows.

$$
e_{\text {tot }}=\sum_{i=1}^{n} \operatorname{part}_{\text {source }} \cdot E_{\text {tot }} \cdot e_{\text {source }}
$$

- $\quad e_{\text {tot }}$ is total emission (or the emission coefficient);

- $\quad n$ is the number of different energy sources;

- And part $_{\text {source }}$ is the fraction of the total amount of energy obtained from a specific energy source;

- $\quad E_{t o t}$ is total amount of energy consumed;

- $e_{\text {source }}$ is the specific emissions from energy sources.

In this study an analysis of these conversion factors for energy generation presented by different databases has been executed. The following energy sources are distinguished: LPG, Diesel, Electricity, Coal, Oil and Natural Gas. To illustrate the importance of the source of energy some specific emissions are given (Guidelines to Defra, 2009).

- Coal $0,31 \mathrm{~kg} \mathrm{CO}_{2}$ per produced $\mathrm{kWh}$;

- $\quad$ LPG $0,21 \mathrm{~kg} \mathrm{CO}_{2}$ per produced $\mathrm{kWh}$;

- $\quad$ Oil (fuel) $0,26 \mathrm{~kg} \mathrm{CO}_{2}$ per produced $\mathrm{kWh}$;

- $\quad$ Natural Gas kg 0,18 $\mathrm{kg} \mathrm{CO}$ per produced $\mathrm{kWh}$;

- Electricity (UK) $0,27 \mathrm{~kg} \mathrm{CO}_{2}$ per produced $\mathrm{kWh}$.

All energy consumptions in this study are assumed to be grey. Grey energy is all the energy that is generated from fossil fuel. Green energy is that energy that is generated with sustainable methods, like wind and solar energy. Where deviation occurred in the given dimensions, conversions were done with gross fuel properties provided in the Defra database, presented in Table 3.

\begin{tabular}{|l|l|l|l|l|}
\hline \multicolumn{2}{|l|}{ Table 3. Fuel Properties (Defra, 2009) } \\
\hline & Net Caloric Value & Density & Density & Net Caloric Value \\
\hline & GJ/tonne & $\mathrm{kg} / \mathrm{m}^{3}$ & Litres/tonne & $\mathrm{kWh} / \mathrm{kg}$ \\
\hline LPG & 46,98 & 508,1 & 1968 & 13,05 \\
Diesel & 43,27 & 834,0 & 1199 & 12,02 \\
Coal & 25,56 & - & - & 7,10 \\
Oil & 41,46 & 986,2 & 1014 & 11,52 \\
Gas & 47,59 & 0,7459 & 1340651 & 13,22 \\
\hline
\end{tabular}


The evaluated figures originate from online published databases. The consulted databases are:

- WRAP, Waste and Resources Action Program (UK, 2006)

- GHG, Greenhouse Gas Protocol (IPCC, USA, 2004)

- Prorail, De $\mathrm{CO}_{2}$-prestatieladder (NL, 2009)

- Guidelines to Defra (UK, 2009)

- PCC Project Carbon Calculating (NL, 2009)

Energy transformation factors are crucial in the determination factors for a product. The variety in these factors used in five different databases is presented in the Figure 3. Not every database presents a figure for each energy source, so the amount of data differs per source. They are the basis of each emission coefficient. Logically deviations in these specific energy emission coefficients cause deviations in the emission coefficients of all emissions deducted from these figures.

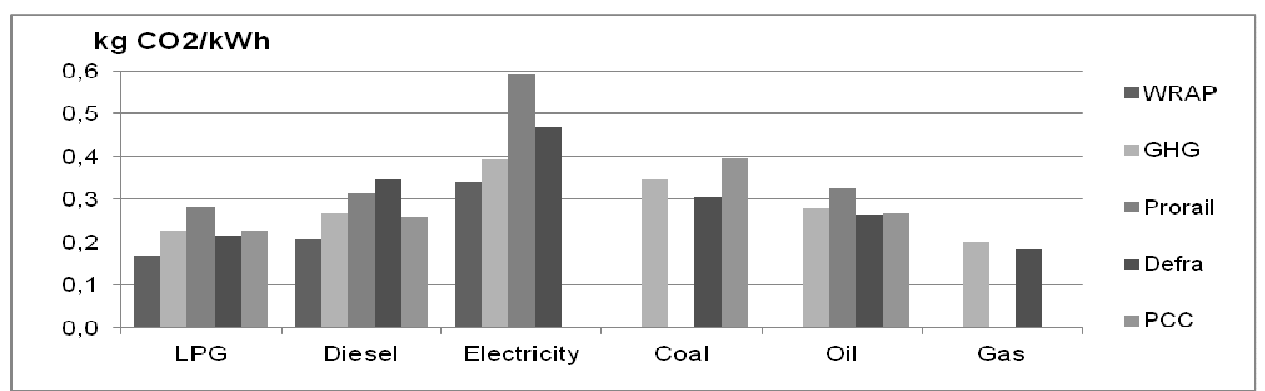

Figure 3 Variations in emission coefficients for energy (The WRAP database provides $\mathrm{CO}_{2} \mathrm{e}$ emissions. These are converted to $\mathrm{CO}_{2}$ emissions by reducing them by a factor of 0,79 )

From the figure it becomes clear that even if the amount of consumed energy and the sources from which that energy was generated are fixed, the outcome of the embodied carbon can differ from database to database. In order of magnitude the amounts are corresponding, but there are differences.

Generally the WRAP database, the Greenhouse Gas Protocol and the figures from Defra show large coherence. Prorail generally gives a bit higher values, especially for electricity. The Project Carbon Calculating tool seems to alter between higher and lower values than the others.

The differences make a combination in data from various sources difficult and not to be recommended.

\section{Incoherence in databases}

Not every database contains the same information on the same subject. Different causes can be assigned for this phenomenon:

1. The options chosen for recycling (not applicable on energy generation).

2. The judgment of environmental impacts. Databases can either present the carbon emission in raw figures or attribute a weighted value to an emission. The Kyoto protocol for instance was based on Carbon Dioxide equivalent $\left(\mathrm{CO}_{2} \mathrm{e}\right)$ values and expresses all greenhouse gasses in one figure. Similar to the $\mathrm{CO}_{2} \mathrm{e}$ values the Intergovernmental Panel on Climate Change (IPCC) credits emissions on Global Warming Potentials (GWP). A GWP is an indicator that reflects the relative effect of greenhouse gas in terms of climate change considering a fixed time period such as 100 years (GWP100).Several other methods exist where different emissions get credited in different manners. Usually by using a point system emissions to the air can for instance be assigned more points than pollution to the water.

3. The age of the database. Generally production processes become more efficient in the course of time, so emissions for a product reduce.

4. The origin of the database. The location where the database was assembled is important because it determines which production processes are regarded during the setup.

5. Different details of the end product. The definition of a product or service can be interpreted in different ways causing different emissions. 


\section{CONCLUSIONS}

\section{Case study}

Despite of the complications presented in the previous paragraph a calculation of the Carbon Footprint for the various designs of the reinforcement at the coast of Scheveningen has been made using multiple data sources. In Table 4 the results of the Carbon Footprints are given in the last column under the 'Cradle-to-Grave' principle. The other scopes are also presented.

\begin{tabular}{|l|l|l|l|}
\hline \multicolumn{5}{|l|}{ Table 4. Embodied Carbon for all variants } \\
\hline Variant & 'Cradle-to-Gate' & 'Cradle-to-Site' & 'Cradle-to-Grave' \\
\hline & EC (kg)/m & EC $(\mathrm{kg}) / \mathrm{m}$ & EC $(\mathrm{kg}) / \mathrm{m}$ \\
\hline Beach nourishment & 666 & 2682 & 7483 \\
Concrete columns & 6538 & 7316 & 8992 \\
Rock & 2223 & 3157 & 4833 \\
Asphalt & 2780 & 3105 & 4781 \\
Elastocoast & 2672 & 3033 & 4709 \\
\hline
\end{tabular}

The Carbon Footprint and the distinction between the different scopes are presented in Figure 4. 'Cradle-to-Grave' entails for all hard constructions an amount $400 \mathrm{~m}^{3} / \mathrm{m}$ of nourished sand. Without this additional amount of maintenance the beach nourishment variant would have the largest Carbon Footprint.

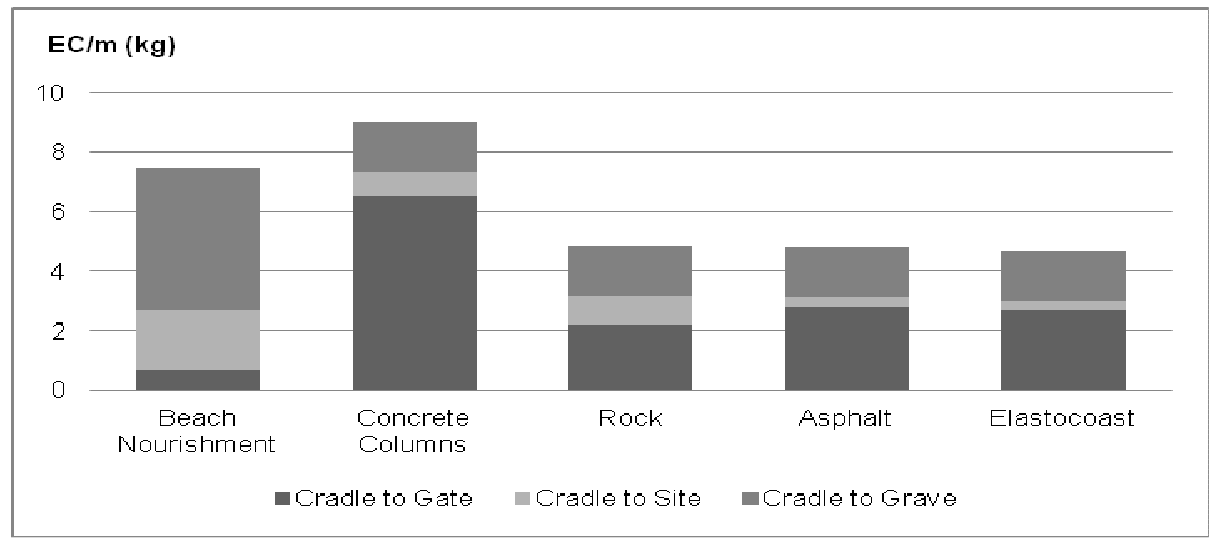

Figure 4 Carbon Footprints for three different stages (scopes) of the evaluated variants

Five observable conclusions can be drawn from Table 4 and Figure 4.

1.Most important for the determination of the Footprint is the material used for the different alternatives. Transport and placing activities have shown to be quite similar for all designs as well are the maintenance activities.

2.The exception on these conclusions is the beach nourishment. For this alternative the maintenance activities are very important and make up the largest part of the total. This is the main reason why the largest emissions are found for the soft solution. Elastocoast, asphalt and the natural rock revetment all have comparable lower footprints.

3.From the hard solutions concrete causes the largest carbon emission, mainly due to the production process of the cement clinker. The dimensions of the top layers are very determining for the emissions and ranking of the designs.

4.Elastocoast takes the highest position for emissions with respect to weight $(0,117 \mathrm{~kg} \mathrm{CO} / \mathrm{kg})$ and the emissions for rock and concrete columns are comparable and relatively low (respectively $0,056$ and $0,058 \mathrm{~kg} \mathrm{CO} 2 / \mathrm{kg})$, a kilogram of asphalt lies in the middle $\left(0,072 \mathrm{~kg} \mathrm{CO}_{2} / \mathrm{kg}\right)$. However for the Elastocoast design a smaller layer thickness for the top layers is required.

5. The most important conclusion to be drawn from this research is that it is possible to compose a ranking of coastal protection systems and differences between in carbon emissions for the various designs become clear by applying the method.

\section{Emissions}

Just as important however are the uncertainties in emission values. For many materials a large range of emissions can be found in different databases. This is caused by a number of reasons, but the three most important aspects influencing the emission figures are: the total amount of consumed energy, 
the distribution of this amount of consumed energy over different energy sources and the emissions linked to the generation of energy from a specific source.

Other reasons for deviations in emission values can lie in the supporting information provided by a database. A clear description of the actual emission and the processes that are incorporated (direct/indirect) can reduce the width of the range of available data.

A result from the previous statement is that data from different databases is not automatically compatible. Some databases stick to a point system rather than presenting actual emissions. The scope of the emission can cause additional incompatibility. If for instance a 'Cradle-to-Site' scope is applied no additional emissions for transport have to be accounted for.

\section{Future use}

The following critical notes should be made when using the Carbon Footprint in future studies.

1. Applying the Carbon Footprint as a means of determining the environmental impact has proven to be quite effective in the comparison of the different stages of the construction and the different components of a design. The structure as a whole can be considered as well as solely the top layer materials or transport contributions. It is an accessible way for governments to control its pollution and keep track of different sectors.

2. Direct link to the used amount of Energy and the $\mathrm{CO}_{2}$ emissions connected to that amount. The amount of Energy is a more reliable value than $\mathrm{CO}_{2}$ emissions because of differences in databases in conversion factors. However greenhouse gasses are more tangible and practical than amounts of energy, because of intergovernmental agreements.

3. The determination of the Carbon Footprint is a case study on one project cannot be projected on other similar projects because of differences in for instance transportation distances. However when a set of footprints is determined for several projects a more general statement can be made as to the preferable manners of coastal protection in terms of sustainability.

4. The negative aspects of the Carbon Footprint are the fact that it merely focuses on air pollution by greenhouse gas emission. This does not represent an all-round view of a service of product pollution. Aspects of a project and its environmental impact that only become clear if a larger scope than the lifetime of a product is regarded, are not always represented properly. Waste reduction and reuse are examples of these aspects.

\section{REFERENCES}

Bruce T.\& Chick J. (2009) Energy and Carbon Costing of Breakwaters, University of Edinburgh

Centre for Sustainability (C4S), Waste and Resources Action Programme WRAP (2006), The $\mathrm{CO}_{2}$ emissions estimator tool for the use of aggregates in construction, ISBN: 1-84405-268-0

Greenhouse Gas Protocol (2004), www.ghgprotocol.org, World Resources Institute and World Business Council for Sustainable Development, USA, ISBN 1-56973-568-9

Guidelines to Defra / DECC's GHG Conversion Factors for Company Reporting (2009), AEA for the department of Energy and Climate Change (DECC) and the department for Environment, Food and Rural affairs (Defra)

Hammond G. \& Jones (2008), Inventory of Carbon \& Energy (ICE) version 1.6a, Sustainable Energy Research Team (SERT), Department of Mechanical Engineering, University of Bath, UK

Hendriks Ch.F. (2001) Sustainable Constructions, Aeneas, Boxtel, ISBN 90-75365-45-8

Project Carbon Calculator PCC versie 2.0(2009), Koninklijke BAM Groep nv

Prorail (2009), De $\mathrm{CO}_{2}$ - Prestatieladder / Het certificeren, Prorail / AKI 